\title{
CARACTERIZAÇÃO DOS PACIENTES COM SÍNDROME DE GUILLAIN-BARRÉ INTERNADOS EM UM HOSPITAL UNIVERSITÁRIO
}

\author{
Alvana Moraes*, Ana Cristina Geiss Casarolli**, Thais Dresh Eberhardt**, Lili Marlene Hofstatter*** \\ Autora correspondente: Aluana Moraes - aluanamoraes@hotmail.com \\ * Especialista em Gerenciamento de Enfermagem em Clínica Médica e Cirúrgica, Mestranda do Programa de Enfermagem \\ da Universidade Estadual de Londrina \\ ** Especialista em Gerenciamento de Enfermagem em Clínica Médica e Cirúrgica, Mestranda do programa de pós- \\ graduação em Enfermagem da Universidade Federal de Santa Maria \\ *** Professora Mestre do Departamento de Enfermagem da Universidade Estadual do Oeste do Paraná e do Programa de \\ Residencia de Gerenciamento de Enfermagem em Clinica Médica e Cirúrgica da Universidade Estadual do Oeste do Paraná
}

\section{Resumo}

O presente estudo tem por objetivo caracterizar clinicamente os pacientes diagnosticados com Síndrome de Guillain-Barré internados no Hospital Universitário do Oeste do Paraná, no período de o1 de janeiro de 2009 a 31 de dezembro de 2012. Este estudo se caracteriza como quantitativo, descritivo, retrospectivo e documental. Foram identificados oito pacientes com Sindrome de GuillainBarré, no período de 2009 a 2012, esses foram caracterizados quanto as características clinicas. Os resultados identificaram que os pacientes investigados no período, 4 (50\%) eram do sexo masculino e 4 (50\%) eram do sexo feminino. O estudo possibilitou identificar que a Síndrome de Guillain-Barré é uma doença que pode atingir tanto o sexo feminino, quanto o sexo masculino em sua porcentagem igualitária. Seus sintomas mais característicos consistem em formigamento em membros superiores e membros inferiores e parestesia distal em membros inferiores. O conhecimento das características dos pacientes internados que apresentam a Síndrome de Guillain-Barré, possibilitando identificar os aspectos da doença e suas particularidades.

Palavras-chave: Síndrome de Guillain-Barré; Característica; Hospital. 


\title{
CHARACTERISTICS OF PATIENTS WITH SYNDROME GUILLAIN-BARRÉ HOSPITALIZED IN A UNIVERSITY HOSPITAL
}

\begin{abstract}
This study aims to clinically characterize patients diagnosed with Guillain-Barré syndrome admitted to the University Hospital of Western Paraná, in the period from 1 January 2009 to 31 December 2012. This study is characterized as quantitative, descriptive, retrospective documentary. Eight patients with Syndrome GuillainBarré were identified from 2009 to 2012 , these were characterized the clinical features. The results showed that patients investigated in the period, 4 (50\%) were males and 4 (50\%) were female. The study identified that the Guillain-Barré syndrome is a disease that can achieve both female, as males in their equal percentage. His most characteristic symptoms consist of tingling in upper and lower limbs and distal paresthesia in the lower limbs. The knowledge of the characteristics of hospitalized patients with the Guillain-Barré syndrome, making it possible to identify the aspects of the disease and its peculiarities.
\end{abstract}

Keywords: Guillain-Barré syndrome; Feature; Hospital.

\section{INTRODUÇÃO}

A Síndrome de Guillain-Barré pode ser classificada com uma polineuropatia aguda, desmielinizante e inflamatória de caráter auto imunológico. ${ }^{(1)}$ Caracteriza-se por uma desmielinização dos nervos motores principalmente, mas pode também atingir os nervos sensitivos, ocorrendo um comprometimento periférico ascendente, progressivo e simétrico. ${ }^{(2)}$

Sua causa exata ainda encontra-se desconhecida, no entanto alguns estudos demonstram a correlação com infecções e reações vacinais. Segundo alguns autores ${ }^{3}$ as infecções do trato respiratório superior e infecções gastrointestinais como diarreia associada à bactéria Campylobacterjejuni, são as maiores causas relacionadas com a doença. Ainda infecções virais como citomegalovírus, vírus do Herpes, da hepatite A e B e vírus da AIDS estão associadas em menor proporção. ${ }^{(3)}$

Sua manifestação clinica caracterizam-se pela dor e perda de força muscular progressiva dos membros inferiores, evoluindo para a diminuição ascendente dos movimentos. Podem ocorrer sintomas sensitivos como o formigamento e sensação de queimação nos membros inferiores e superiores de forma distal. Ainda a doença pode evoluir para paralisia flácida, afetando músculos da face, orofaringe, respiração de deglutição. ${ }^{(3)}$

O diagnóstico ocorre primeiramente de forma clínica, na qual o paciente apresenta dificuldade para deambulação, sensação de dormência ou fraqueza na musculatura, sensação de perda de força na musculatura esquelética, em alguns casos intensa dor em toda a extremidade corpórea. Posteriormente, para a confirmação do quadro clínico deve-se realizar o diagnóstico laboratorial, que pode ser através da análise do líquido cefalorraquidiano ou através do diagnóstico eletrofisiológico, também conhecido como eletroneuromiografia.

O tratamento para a Síndrome de Guillain-Barré pode ocorrer através de corticosteroides, imunoglobulina humana intravenosa e plasmaferese. ${ }^{(4)}$ 
No entanto a terapia de maior escolha e com meIhor resultado é a imunoglobulina humana intravenosa, na qual dever ser administrada $0,4 \mathrm{~g} / \mathrm{Kg} /$ dia, por via intravenosa durante cinco dias consecutivos. ${ }^{(4)}$

O presente estudo tem por objetivo caracterizar clinicamente os pacientes diagnosticados com Síndrome de Guillain-Barré internados no Hospital Universitário do Oeste do Paraná, no período de 01 de janeiro de 2009 a 31 de dezembro de 2012.

\section{METODOLOGIA}

Para instrumentalizar e viabilizar o alcance do objetivo proposto, este estudo se caracteriza como quantitativo, descritivo, retrospectivo e documental. Segundo alguns autores ${ }^{(6)}$ a pesquisa descritiva tem como objetivo primordial a descrição das características de determinada população ou fenômeno ou o estabelecimento de relações entre variáveis.

A população do estudo constituiu-se de todos os pacientes que foram diagnósticos com Síndrome de Guillain-Barré, entre o período de or de janeiro de 2009 a 31 de dezembro de 2012. A amostra foi conformada através do seguinte critério de inclusão: idade maior ou igual a 18 anos, ou, com a presença de acompanhante responsável no caso de idade inferior, que aceitasse participar da pesquisa. Foram excluídos do estudo todos os pacientes que não aceitaram participar do estudo e que foram diagnósticos fora do período da pesquisa.

Para a coleta dos dados utilizou-se um instrumento intitulado "caracterização dos pacientes" que continha as seguintes variáveis: sexo, idade, sintomas, doença prévia, exames de diagnóstico, tratamento, complicações, evolução, no período de O1 a 31 de março de 2013.

Os dados foram coletados no prontuário eletrônico do Hospital Universitário do Oeste do Paraná, através do prontuário eletrônico do pacien-

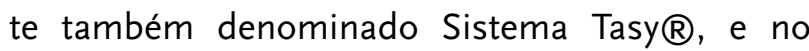
Serviço de Arquivo Médico (SAME) da instituição hospitalar. Para a análise dos dados, as respostas foram digitadas em um banco de dados em planiIhas do Microsoft Excel. Os dados serão agrupados e dispostos usando-se a estatística simples descritiva.

A coleta de dados somente foi realizada após análise e aprovação do Comitê de Ética em Pesquisa (CEP) da Universidade Estadual do Oeste do Paraná (UNIOESTE), através do parecer $n^{\circ} 413.915 / 2013$.

\section{RESULTADO E DISCUSSÃO}

Foram identificados oito pacientes com Sindrome de Guillain-Barré, no período de 2009 a 2012, esses foram caracterizados quanto as características clinicas.

Os resultados identificaram que os pacientes investigados no período, 4 (50\%) eram do sexo masculino e 4 (50\%) eram do sexo feminino. Os indivíduos do sexo masculino possuem risco maior de desenvolvimento da Síndrome de Gullain-Barré, do que os indivíduos do sexo feminino.(7) No entanto os pacientes identificados na pesquisa apresentaram equivalência igual entre os sexos, em um determinado período.

Com relação a faixa etária verificou-se que 1 $(12,5 \%)$ possuía idade menor que cinco anos de, 1 $(12,5 \%)$ paciente possuía idade menor que 20 anos, $3(37,5 \%)$ pacientes possuíam entre 20 a 30 anos, $1(12,5 \%)$ paciente possuía entre 40 a 49 anos, 2 (25\%) pacientes possuíam entre 50 a 60 anos de idade. A incidência anual da Síndrome de Guillain-Baré é 1 a 4 por 100 mil habitantes, com idade entre 20 a 40 anos, no Brasil ${ }^{4}$. Estudo realizado no ocidente possui frequência do desenvolvimento da doença em adultos, do que em crianças, o que é possível identificar por meio da pesquisa que somente dois indivíduos possuem idade menor que $20(25 \%)$ anos, e os demais indivíduos possuíam idade acima de $20(75 \%)$ anos, sendo considerados adultos. ${ }^{(7)}$ 
Com relação aos sintomas encontrados durante o período de avaliação para internamento hospitalar todos (100\%) os pacientes da pesquisa apresentaram formigamento em membros superiores (MMSS) e membro inferiores (MMII), parestesia distal em MMII. Ainda 1 (12,5\%) paciente apresentava diarreia e cefaleia. Os pacientes acometidos pela Síndrome de Guillain-Barré apresentam manifestações clinicas de paralisia motora com evolução rápida, pode ou não apresentar alterações sensoriais, sensação de peso e dormência dos membros inferiores, fraqueza, formigamento dos MMSS e MMII, com inicio pelos MMII. Além disso, o individuo pode apresentar comprometimento dos nervos cranianos inferiores e região bulbar, evoluindo para insuficiência respiratória. ${ }^{(7)}$

No que se refere as doenças previas encontradas nos participantes da pesquisa foram: hérnia discal $(12,5 \%)$, diarreia constante de causa não investigada $(12,5 \%)$, infecção do trato urinário recorrente $(12,5 \%)$, hipercolesterolemia $(12,5 \%)$, hipertensão $(12,5 \%)$, paralisia flácida $(12,5 \%)$, síndrome do intestino irritável (12,5\%), febre reumática (12,5\%), infecção gastrointestinal (12,5\%). Para alguns autores o desenvolvimento da doença pode estar relacionado com quadro infeccioso agudo de origem respiratória ou gastrointestinal, que ocorra por 1 a 3 semanas. ${ }^{(7)}$

Para o diagnostico da doença é necessário a investigação clinica definida pelo reconhecimento do padrão de paralisia rapidamente progressivo com arreflexiva, associado aos exames laboratoriais (exame de líquor), e exames de eletrofisiologia. Para o exame de líquor, deve ser encontrado nível de proteína elevado, entre 1 a $10 \mathrm{~g} / \mathrm{L}(100$ a 1000 $\mathrm{mg} / \mathrm{dL}$ ), geralmente 48 horas após os sintomas da doenças estarem presentes. Para o exame de eletrofisiologia, é utilizado a eletroneuromiografia, o paciente deve apresentar ondas que indiquem a redução proximal da velocidade de condução nervosa. ${ }^{(7)}$ Os pacientes (100\%) do estudo foram diagnosticados com a doença por meio do exame de líquor e pela eletroneuromiografia, somente um paciente realizou ressonância magnética para excluir outras causas.
Para o tratamento da doença, estudos indicam que deve ocorrer por meio da imunoglobulina in travenosa (IgIV) em altas doses ou a plasmaférese, pois possuem igualmente resultados satisfatórios. ${ }^{(7)}$ Já para o Ministério da Saúde o tratamento da Síndrome de Guillain-Barré pode ocorrer com corticosteroide, imunoglobulina humana e plasmaférese. ${ }^{(4)}$ Para o uso de imonoglobulina humana faz-se necessário um esquema que seja de 0,4 $\mathrm{g} / \mathrm{Kg} /$ dia por via intravenosa, por um período de cinco dias, para o uso da plasmaférese faz-se importante o uso de $200-250 \mathrm{ml} / \mathrm{kg}$ a cada 48 horas variando conforme o grau da doença. (4) Todos os indivíduos da pesquisa utilizaram o tratamento por meio da Imunoglobulina Humana, o que demonstra a importância do tratamento por meio dessa medicação.

Com relação as complicações desenvolvidas pela doença apenas um paciente obteve insuficiência respiratória, resultando em ventilação mecâni$\mathrm{ca}$, internação em unidade de terapia intensiva e posteriormente óbito, com tempo de internação de seis meses de internação. Os demais pacientes não apresentaram complicações, evoluindo para alta hospitalar, permanecendo internados por um período de trinta dias. Esse período de internação varia devido a complexidade do diagnostico da doença e do processo de tratamento estabelecido pela instituição.

\section{CONSIDERAÇÕES FINAIS}

Por apresentar patologia complexa e de incidência crescente, tal trabalho abrangeu as características clinicas dos pacientes com Síndrome de Guillain-Barré, possibilitando confirmar os estudos previamente realizados e distinguir as especificidades dos indivíduos participantes do estudo.

O estudo possibilitou identificar que a Síndrome de Guillain-Barré é uma doença que pode atingir tanto o sexo feminino, quanto o sexo masculino em sua porcentagem igualitária. Sua faixa etária é predominantemente de 20 a 30 (37,5\%). Seus sintomas mais característicos consistem em formigamento 
em membros superiores e membros inferiores e parestesia distal em membros inferiores.

As doenças previas mais encontrados nos participantes do estudo consistem em hérnia discal, diarreia constante de causa não investigada, infecção do trato urinário recorrente, hipercolesterolemia, hipertensão, paralisia flácida, síndrome do intestino irritável $(12,5 \%)$, febre reumática, infecção gastrointestinal.

Com relação aos exames para diagnóstico todos os pacientes do estudo, foram diagnóstico por meio líquor e pela eletroneuromiografia. O tratamento de escolha ocorreu por meio da Imunoglobulina Humana em todos os participantes da pesquisa. Entre as complicações encontradas pela doença apenas um paciente desenvolveu insuficiência respiratória, evoluindo para uso de ventilação mecânica.

Considera-se uma limitação do estudo a inexistência de diagnóstico médico nos prontuários dos pacientes, impossibilitando a investigação do estudo. Todavia, é de importância salientar que as informações sobre a Síndrome de Guillain-Barré são escassas no que tange a etiologia, prevenção e formas de tratamento.

Através do presente estudo e do conhecimento das características dos pacientes internados que apresentam a Síndrome de Guillain-Barré, foi possível identificar os aspectos da doença, bem como suas particularidades.

\section{REFERENCIAS}

1. Volquind D, Fellini RT, Rose GL, Tarso GP. Anesthesia for cesarean section in a patient with Guillain-Barre syndrome: case report. Rev. bras. anestesiol. 2013. [cited 2014 July 26]; 63(4):369-371. Available from: http://www.scielo.

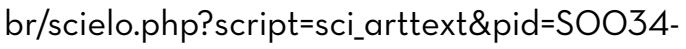
70942013000400012\&lng=en

2. Bolan RDS, Bó KD, Vargar FR, Moretti GRF, Almeida LPD, Almeida GKPD, et al. Síndrome de Guillain-Barré. Rev. AMRIGS. 2007. [acesso 15 maio 2014];51(1):58-61. Disponível em: http:// www.amrigs.com.br/revista/51-O1/rcO3.pdf

3. Beneti GM, Silva DLD. Síndrome de GuillainBarré. Semina cienc. biol. saúde. 2006 [acesso 14 mai 2014];27(1):57-69. Disponível em: http:// www.uel.br/revistas/vel/index.php/seminabio/ article/view/3529

4. Brasil. Ministério da Saúde. Protocolo clínico e diretrizes terapêutica Síndrome de Guillain Barré. 2009 [acesso 14 maio 2014]. Disponível em: http://www.vigilanciaemsaude.ba.gov.br/ sites/default/files/galeria/texto/2015/O6/19/ pcdt-sindrome-guillain-barre-livro-2009.pdf

5. Haldeman D, Zulkosky K. Treatment and nursing care for a patient with Guillain-Barré syndrome. Dimens. crit. care Nurs. 2005. [cited 2014 may 28]; 24(6):267-272. Available from: http://journals.lww.com/dccnjournal/ Abstract/2005/11000/Treatment_and_Nursing_ Care_for_a_Patient_With.4.aspx

6. Gil AC. Como elaborar projetos de pesquisa. $4^{\mathrm{a}}$ ed. São Paulo: Atlas; 2002.

7. Longo DL, et al. Medicina Interna de Harrison. $18^{\mathrm{a}}$ ed. Porto Alegre: AMGH; $2 \mathrm{O} 13$. 


\title{
REALIZAÇÃO DA SOROLOGIA PARA HIV NO PRÉ-NATAL \\ - conhecimento e percepção da gestante •
}

\author{
Raquel Silveira Mendes Correio*, Melânia de Albuquerque Andrade Lima Correio**, Cristiane \\ Santiago Natário Branco Correio**, Ysabely de Aguiar Pontes Pamplona Correio*** \\ Endereço para correspondência: Jose dos Campos. E-mail: ysabelypontes@hotmail.com \\ * Universidade Estadual do Ceará. Enfermeira. Especialista em Saúde Pública pela Universidade Estadual do Ceará. \\ Mestranda em Cuidados Clínicos na Universidade Estadual do Ceará \\ ** Universidade Estadual do Ceará. Enfermeira. Especialista em Saúde Pública pela Universidade Estadual do Ceará \\ *** Universidade Católica de Santos. Enfermeira. Doutoranda em Saúde Coletiva da Universidade Católica de Santos. \\ Bolsista CAPES. Integrante do Núcleo de Estudos e Pesquisas em Condições Sociais e Saúde
}

\section{Resumo}

A pesquisa apresentou como objetivo avaliar o conhecimento e a percepção das gestantes durante o acolhimento pré-natal realizado em um hospital-maternidade público situado em Fortaleza-Ceará, em relação à realização da Sorologia para HIV. Trata-se de uma pesquisa com abordagem qualitativa. Foram selecionadas 10 gestantes. As informações foram coletadas nos meses de março e abril de 2009, através da aplicação de entrevista. As informações foram analisadas e correlacionadas com a literatura vigente. A maioria das gestantes reconheceu a importância da realização do pré-natal, da necessidade de prevenir a transmissão vertical do HIV na gestação e da realização da Sorologia para o HIV na gestação. Porém, alguns conceitos referentes à infecção e a realização da Sorologia para o HIV foram aprendidos incorretamente, bem como a maioria das gestantes não incorporou de forma efetiva e rotineira as práticas preventivas relacionadas à transmissão do HIV inclusive verticalmente. Palavras-chave: Cuidado Pré-Natal; Promoção da Saúde; HIV.

\section{REALIZATION OF HIV TESTING IN PRENATAL CARE}

\author{
-knowledge and perception of pregnancy•
}

\begin{abstract}
The main objective of the research is to evaluate the knowledge and perception of pregnant women during prenatal care performed in a public maternity hospital in Fortaleza-Ceará, in relation to the implementation of HIV serology. This is a qualitative research. Ten women were selected for this study. Data were collected in
\end{abstract}


March and April 2009, through an interview application interview. The data were analyzed and correlated with the current literature. Most women recognized the importance of completing the pre-natal, the need to prevent vertical transmission of HIV during pregnancy and the need of HIV serology during pregnancy. But some concepts related to infection and the accomplishment of the test results for HIV were improperly seized, and most women were not incorporated effectively and routinely to the preventive practices related to HIV transmission including vertically.

Keywords: Prenatal Care; Health Promotion; HIV.

\section{INTRODUÇÃO}

O Pré-Natal tem como finalidade oferecer assistência materno-infantil qualificada e humanizada durante os períodos da gestação, do parto e do puerpério, sendo fundamental para assegurar a saúde materno-infantil.

No Brasil, o início do processo de implantação das ações em saúde relacionadas à assistência materno-infantil está historicamente relacionado à criação, em 1953, do Ministério da Saúde, tendo sido efetivado durante a década de 70 , quando foram estabelecidos programas de acompanhamento nutricional da gestante, de assistência à gravidez, ao parto e ao puerpério, e de estímulo ao aleitamento materno. Em associação ao desenvolvimento destes programas, o Ministério da Saúde criou, em 1978, o Programa de Prevenção da Gravidez de Alto Risco.

Outro marco importante relacionado à política nacional de atenção integral a saúde da materno-infantil desenvolvida pelo ministério da Saúde refere-se à implantação, em 1984, do Programa de Assistência Integral à Saúde da Mulher (PAISM), através do qual foram contempladas ações básicas de saúde relacionadas ao planejamento familiar, a ampliação, a qualificação e a humanização da atenção ginecológica e obstétrica (compreendendo todo o ciclo vital feminino), bem como a prevenção e o controle das Doenças Sexualmente Transmissíveis (DST), o que incluía, também, a prevenção da transmissão do Vírus da Imunodeficiência Humana (HIV).
A evolução da epidemia provocada pela transmissão do Vírus da Imunodeficiência Humana (HIV), no Brasil, afeta mulheres de todos os segmentos sociais. Entretanto, mostram-se freqüentemente mais vulneráveis as mulheres menos favorecidas do ponto de vista sócio-econômico, com profundas implicações para as ações de prevenção e de assistência. ${ }^{(1)}$

No Brasil, durante o período compreendido entre 1980 a 2006 , foram notificados 433.067 casos de infecção pelo HIV dos quais $67,2 \%$ dos casos foram diagnosticados em homens e $32,8 \%$ casos foram diagnosticados em mulheres. ${ }^{(2)}$

O aumento do número casos de mulheres contaminadas pelo Vírus da Imunodeficiência Humana podem ser considerados como o reflexo dos aspectos culturais que permeiam a educação sexual e o desenvolvimento da sexualidade feminina. Em razão do crescente número de mulheres infectadas em idade fértil, vem-se observando que, atualmente, a quase totalidade de casos de contaminação pelo HIV, em menores de 13 anos, acontece através da transmissão vertical.(3)

Denomina-se transmissão vertical a situação em que a criança é infectada pelo Vírus da Imunodeficiência Humana durante a gestação (35\% dos casos), o parto ( $65 \%$ dos casos) ou a amamentação (7\% até $22 \%$ por exposição/mamada). ${ }^{(2)}$

Apesar do alto índice de cobertura pré-natal no Brasil, parte das gestantes deixa de submeter-se 
ao exame laboratorial que diagnostica a presença do Vírus da Imunodeficiência Humana no organismo (conhecido como Sorologia para HIV), seja por dificuldade de acesso e /ou pela qualidade da atenção, constituindo uma das principais barreiras para redução da transmissão vertical deste vírus.

O Ministério da Saúde instituiu o Programa de Humanização no Pré-natal e Nascimento (PHPN), através da Portaria GM No. 569 de 01/06/2000 estabelecendo que para o adequado acompanhamento do pré-natal e da assistência à puerperal, o serviço de saúde deve realizar a primeira consulta do pré-natal até $\circ 4^{\circ}$ mês de gestação, bem como garantir a realização de no mínimo 06 consultas sendo preferencialmente or consulta no $1^{\circ}$ trimestre, or consulta no $2^{\circ}$ trimestre, 03 consultas no $3^{\circ}$ trimestre, e or consulta no puerpério (até 42 dias após o nascimento).

Na primeira consulta todas as informações sobre o histórico de ocorrências de doenças desenvolvidas pela gestante e/ou família devem ser fornecidas, e a mulher pode aproveitar o momento para expor todas as suas dúvidas sobre as transformações do seu corpo relacionadas à gestação.

Serão solicitados exames laboratoriais, com o objetivo de detectar alterações na condição de saúde materna que possam afetar a saúde do binômio mãe-bebê e o bom andamento da gestação, tais como: hemograma; sorologias para síflis (VDRL, Or exame na $1^{a}$ consulta e o outro próximo à trigésima semana da gestação), rubéola, toxoplasmose, e HIV; glicemia; grupo sanguíneo e fator Rh; sumário de urina (Or exame na $1^{\text {a }}$ consulta e o outro próximo à trigésima semana da gestação), parasitológico de fezes, assim como outros. Esses exames serão repetidos no decorrer da gestação para a confirmação do estado de saúde da gestante e do bebê, ou quando o profissional julgar necessário.

Outros exames também poderão ser solicitados. É imprescindível avaliar a imunidade desta gestante em relação à aplicação de doses da vacina antitetânica, para evitar a ocorrência de casos de Tétano Neonatal.
Portanto, a assistência pré-natal é considerada concluída quando estiver registrada a realização das 06 consultas (mínimo), de todos os exames laboratoriais obrigatórios, das doses de vacina necessárias para garantir a imunização contra o Tétano Neonatal, e ainda, de or consulta durante o puerpério. $A$ atenção à gestante terminará após a consulta puerperal.(4)

Informações sobre as diferentes vivências devem ser trocadas entre as mulheres e os profissionais de saúde durante o pré-natal. Essa possibilidade de intercâmbio de experiências e conhecimentos é considerada a melhor forma de promover a compreensão de todas as nuances que envolvem a gestação. ${ }^{(5)}$

Dentro dos princípios gerais e das diretrizes para atenção obstétrica e neonatal, o acolhimento pode ser considerado essencial, pois implica na recepção da gestante, desde sua chegada na unidade de saúde, permitindo que ela expresse seus sentimentos, preocupações e angústias.

Atualmente, o acolhimento é uma estratégia que se insere em vários momentos do atendimento e em diversos contextos do serviço do Sistema Único de Saúde (SUS), incluindo o pré-natal. Este tipo específico de atendimento desempenha um papel importante no diagnóstico precoce da infecção pelo HIV e de outras Doenças Sexualmente Transmissíveis, contribuindo para a promoção da saúde do binômio mãe-bebê. ${ }^{(3)}$

A falta de conhecimento acerca das formas de transmissão do HIV/DST, bem como a indisponibilidade, até o momento, de terapêutica eficaz que permita evitar suas complicações, reforça a necessidade da adoção de medidas preventivas contra estas doenças.

O controle da disseminação da epidemia de HIV/AIDS é um objetivo prioritário do Ministério da Saúde. Considerando a magnitude de suas conseqüências, a implantação de estratégias que visem informar a população sobre as formas de transmissão/prevenção, o diagnóstico (inclusive durante a gravidez) e o tratamento do HIV/AIDS/ DST devem ser consideradas fundamentais para o 
desenvolvimento das intervenções educativas do Enfermeiro e dos demais profissionais que compõem a equipe interdisciplinar de saúde.

As transformações no perfil da epidemia de disseminação do HIV/AIDS demonstram claramente, que a participação da mulher (incluindo a gestante) na cadeia de transmissão desta doença aconteceu de forma progressiva.

Em contra partida, transformações relacionadas à assistência pré-natal também podem ser percebidas. A implantação destas mudanças está relacionada ao processo de descentralização adotado pelo Sistema Único de Saúde, a ampliação da rede de assistência pública à gestante, e a participação popular no desenvolvimento das políticas públicas de saúde.

A manutenção da qualidade de vida do binômio mãe-bebê inclui o atendimento das reais expectativas e necessidades vivenciadas pela mulher $\mathrm{e}$ seu bebê, durante a gestação, parto e puerpério. A equipe interdisciplinar deve estar, durante o acolhimento/consulta pré-natal sensibilizada, mobilizada e capacitada, centralizando esforços com o intuito de oferecer o cuidado baseado nos aspectos antropológicos, psicológicos, sociais e culturais, de forma humana e individualizada.

Apesar da complexidade que envolve o desenvolvimento desta prática, a sua efetivação pode facilitar a sensibilização das gestantes em relação à importância da realização das consultas, a apreensão das informações, bem como a tomada de decisões de forma consciente e responsável referentes à adoção de práticas que auxiliem a manutenção da saúde da mulher e do bebê, e que principalmente, previnam ocorrência de quaisquer patologias, sobretudo a infecção do binômio mãe-bebê pelo Vírus da Imunodeficiência Humana.

Enfim, enfrentar a epidemia do HIV/AIDS nos faz lidar com os aspectos mais essenciais da via humana como à sexualidade, a moral, as relações de gênero, as relações de poder, com a vida e com a morte. A efetivação das práticas preventivas relacionadas à transmissão do HIV (incluindo a transmissão vertical) exige o acúmulo do conheci- mento, a memorização, a contextualização e o envolvimento pessoal da gestante.

Desta forma, os profissionais de saúde que atuam no acolhimento/aconselhamento/consulta pré-natal devem unir esforços para que a sua prática produza, nestas gestantes, o exercício da ampliação do senso de responsabilidade, gerando a mudança de comportamento necessária a prevenção da disseminação do vírus HIV, inclusive verticalmente a partir da realização da Sorologia para HIV durante o pré-natal.

A ideia da construção desta pesquisa surgiu após a realização de estágio das alunas, do curso de Graduação em Enfermagem da Universidade de Fortaleza, em instituições hospitalares públicas localizadas em Fortaleza-Ce, que prestavam atendimento na área Materno-Infantil. Ao término desta visita surgiu o interesse e o desejo de aprofundar conhecimentos acerca do assunto evidenciado, com o objetivo de avaliar o conhecimento e a atitude das gestantes, durante o acolhimento / pré-natal realizado em um hospital-maternidade, situado em Fortaleza-Ceará, em relação à realização da Sorologia para o HIV.

Este estudo objetivou avaliar o conhecimento e a percepção das gestantes, durante o acolhimento pré-natal realizado em um hospital-maternidade público situado em Fortaleza-Ceará, em relação à realização da Sorologia para HIV.

\section{METODOLOGIA}

A pesquisa utilizou abordagem qualitativa e caráter descritivo, permitindo registrar, descrever, analisar e interpretar a natureza atual do tema estudado.

Por meio da pesquisa qualitativa é possível obter o significado dos acontecimentos vividos pelas pessoas, na realidade social, considerando-as como atores sociais, pois, uma das grandes postulações da pesquisa qualitativa é a sua atenção especial pelos pressupostos que servem de fundamentação à vida das pessoas. ${ }^{(6)}$ 
Foi eleito como campo para o presente estudo uma instituição pública localizada em Fortaleza-Ceará, a qual apresentava como um de seus objetivos a assistência/tratamento à gestante e ao bebê, durante os períodos pré-natal, parto e pós-parto.

Este serviço propiciava campo de estágio às diversas universidades e faculdades, contribuindo para o desenvolvimento do ensino e da pesquisa relacionados aos tipos de patologias assistidas/tratadas na instituição, abordando a saúde integral da mãe e da criança. Caracterizou-se, sobretudo, por uma multiplicidade crítica de técnicas e funções, prática interdisciplinar e acessibilidade.

A população escolhida para a realização da pesquisa foi composta por 10 gestantes que realizavam o pré-natal na instituição descrita anteriormente. A identificação das participantes da pesquisa foi pautada em três momentos. Inicialmente, o projeto de pesquisa foi enviado para a Comissão de Ética e Pesquisa da Universidade de Fortaleza. Uma vez aprovado por esta comissão, o projeto de pesquisa foi encaminhado para a instituição mencionada, sendo solicitada à coordenação da instituição a autorização para a sua realização, mediante apresentação de ofícios que elucidaram a importância da pesquisa, seus objetivos e a manutenção do sigilo em relação à identificação das participantes.

Após este momento, foi solicitada a participação das gestantes para a realização desta pesquisa, obedecendo-se o seguinte critério de inclusão: Multípara, que tinha realizado pelo menos uma consulta de acompanhamento pré-natal, antecessora à gestação atual, na instituição selecionada.

A coleta das informações foi realizada nos meses de março e abril de 2009 , sendo utilizadas as seguintes técnicas: Identificação das gestantes que satisfaziam os critérios de inclusão descritos anteriormente e aplicação da entrevista aberta na referida instituição selecionada para a pesquisa.

Durante a análise das entrevistas realizadas com as gestantes escolhidas para participarem desta pesquisa, foram observadas a exaustão e a repetitividade presente nos conteúdos das falas destas mulheres.
A organização e a análise das informações consideraram os seguintes critérios: leitura flutuante das entrevistas aplicadas junto as gestantes, que satisfizeram os critérios determinados pela pesquisa, o que permitiu a construção do corpus, elaboração de categorias que auxiliaram na construção dos primeiros esboços acerca do tema em estudo, exploração do corpus, sendo destacados os trechos mais significativos, incluindo as convergências, as divergências e as idiossincrasias contidas nos depoimentos, ou seja, tudo aquilo que se apresentou com repetitividade em relação ao tema pesquisado e em última instância, foi realizada a etapa referente à dimensão explicativa acerca das informações obtidas durante a realização da pesquisa, relacionando-as com a literatura referente ao tema abordado pelo estudo.

Foi obedecido o que preconiza a Resolução $466 / 12$, quanto aos aspectos éticos da pesquisa com seres humanos:

Eticidade, uma vez que, as pesquisadoras envolvidas comprometeram-se com o máximo de benefícios relacionados ao estudo partindo do princípio de que a compreensão de um fenômeno poderá favorecer a melhoria da atuação profissional da equipe de saúde durante as fases de diagnóstico e tratamento, bem como durante o trabalho de educação em saúde;

Não Maleficência, as pesquisadoras envolvidas ressaltaram a exclusão de atos que poderiam produzir riscos ou desconfortos relacionados à condição biológica, psicológica e social dos sujeitos da pesquisa.

\section{RESULTADOS E DISCUSSÕES}

Os depoimentos das gestantes estavam relacionados ao caráter preventivo do pré-natal relacionado ao risco de adoecimento da mãe, ao oferecimento de exames que verificam o estado de saúde do binômio mãe-bebê, bem como a percepção da possibilidade de transmissão vertical de doenças (incluindo o HIV). A importância do diagnóstico 
(através da realização da Sorologia para HIV) e da adesão ao tratamento específico para o HIV, de forma precoce, também foram alvo da preocupação das gestantes.

A maioria das gestantes ressaltou a importância da realização do pré-natal considerando-o como um recurso fundamental para a prevenção de doenças que podem acometer a gestante e que, principalmente, podem ser transmitidas para o bebê durante a gestação.

Eu acho que fazer o pré-natal é muito importante principalmente para a criança evitar doenças que podem passar da mãe para o filho. E também para evitar que a mãe corra riscos. (Gesta og)

A percepção das complicações advindas do adoecimento da gestante evidenciou a preocupação em realizar os exames solicitados durante o pré-natal. Este fato pode significar que estas muIheres foram sensibilizadas para participarem efetivamente das intervenções relacionadas à manutenção da qualidade de vida da mãe e do bebê, durante o pré-natal.

É bom fazer o pré-natal, né? Porque se a pessoa está grávida e não fizer não sabe se está doente! E no pré-natal a gente faz um monte de exames. (Gesta 08)

Pode influenciar para o bem da saúde da mãe e do bebê. (Gesta 01)

A mulher deve ser preparada para a maternidade através das ações de saúde realizadas durante o pré-natal. Deverão ser conhecidos fatos que podem interferir diretamente na gestação como a história obstétrica anterior, temores mais comuns (da dor, da morte, do esvaziamento, da castração), ou ainda vivências negativas relacionadas à gravidez $\mathrm{e}$ ao parto, que incluem o diagnóstico/transmissão (inclusive vertical) do HIV/DST.

O pré-natal possibilita além do preparo físico da mulher através da realização de exames fundamen- tais, a incorporação da educação na rotina do atendimento, com vistas à promoção da saúde do binômio mãe-bebê..(7)

A maioria das participantes considerou que a realização da sorologia para o HIV durante o pré-natal é fundamental. Os depoimentos consideram que a importância da realização deste exame está vinculada ao diagnóstico e ao inicio do tratamento (precocemente), como também para evitar que o bebê seja contaminado, o que pode influenciar, consideravelmente, na adesão ao tratamento, caso a realização dos dois exames de Sorologia para o HIV apresentem resultados positivos.

Acho que com esse exame eu vou saber se estou contaminada e se corro o risco de passar para o meu bebê. (Gesta 02)

Para poder fazer um tratamento, caso o exame dê positivo. É pra fazer o tratamento pra não passar pro bebê. (Gesta 03)

Descobrindo mais cedo a solução é feita logo. Se demorar pode prejudicar ainda mais o bebê. (Gesta 07)

A oferta do teste anti-HIV durante o pré-natal é fundamental, pois assegura à mulher o direito de receber orientações e medicamentos para o tratamento da infecção pelo HIV, evitando a transmissão vertical deste vírus na maioria dos casos. A redução da transmissão vertical é viabilizada, quando é possível conhecer as gestantes soropositivas para o HIV. Isto impõe aos gestores das políticas públicas de saúde a obrigação de estabelecer estratégias eficazes para o rastreamento da infecção nas gestantes, e assim proteger o bebê quando a mãe está contaminada. ${ }^{(8)}$

Com isto, é possível concluir que o conceito de Transmissão Vertical foi apreendido por estas muIheres, uma vez que, os depoimentos evidenciam que as gestantes reconhecem a possibilidade de transmissão de doenças para o bebê (incluindo o HIV), caso estejam infectadas. Porém, as gestan- 
tes não indicaram em seus depoimentos o conhecimento acerca de todas as formas de transmissão vertical do vírus HIV, uma vez que não houve referência à possibilidade de transmissão vertical durante o parto e/ou amamentação.

O medo referido no discurso de uma das gestantes parece também estar relacionado à preocupação da mãe com o nascimento de um bebê saudável. Em relação ao HIV, o papel materno construído a partir do imaginário social, transfere para a mãe toda a responsabilidade em relação à condição de saúde do bebê.

A gente faz este exame porque a gente sabe como a gente está. Eu me preocupo, tenho medo. (Gesta10)

O objetivo da ação educativa relacionada ao tema HIV/DST é desenvolver no indivíduo a capacidade de analisar criticamente a sua realidade, para que as práticas preventivas sejam inseridas em seu cotidiano.

Existem três tipos de abordagens que permeiam as práticas educativas relacionadas à prevenção do HIV/DST. A primeira utiliza o medo para favorecer a adesão do público-alvo às práticas preventivas; a segunda, é baseada na educação do público-alvo e na sua utilização como multiplicadores do conhecimento preventivo; e a terceira, procura educar o público-alvo com a finalidade de diminuir as situações de risco para assim, promover a prevenção. ${ }^{(4)}$

É possível considerar que a abordagem baseada no medo tenha sido utilizada para favorecer a apreensão do conhecimento relacionado ao HIV/DST ministrado durante o acolhimento/ aconselhamento pré-natal desta gestante.

As equipes de saúde responsáveis pelo acolhimento/aconselhamento/consulta pré-natal utilizam, na maioria das vezes, várias nuances que fazem parte dos três tipos de abordagens utilizados nas práticas educativas relacionadas ao HIV/DST.

A assistência pré-natal reúne um conjunto de procedimentos clínicos e educativos com o objetivo de promover a saúde da gestante e do concepto. ${ }^{(9)}$
$\mathrm{Na}$ maioria das vezes o período de nove meses não é suficiente para que a gestante assimile conhecimento tão variado, em tão poucas consultas. A maioria dos questionamentos trazidos pelas gestantes, embora pareçam elementares para quem escuta, pode representar um problema sério para quem o apresenta. Assim respostas diretas e seguras são fundamentais para garantir o bem-estar da gestante. ${ }^{(7)}$

Contudo, é necessário avaliar até que ponto a educação em saúde baseada na abordagem que inclui o medo está alcançando o seu objetivo primordial, ou seja, o desenvolvimento da prática preventiva do HIV/DST nas gestantes.

A educação deve ser considerada como um ato político, devendo ser utilizada para permitir que o homem seja o sujeito de suas práticas individuais, inseridas em um contexto histórico-social, e em solidariedade com os seus semelhantes. Esta característica conferida ao ato educativo determina que os métodos não devam ser opressores, mas baseados no diálogo e, principalmente, na problematização para que haja crescimento coletivo.

É possível associar as diferentes abordagens educativas, relativas à prevenção do HIV/DST, durante $\mathrm{o}$ acolhimento/aconselhamento pré-natal. Porém, é necessário que os diversos paradigmas inseridos nestas práticas sejam constantemente avaliados (em situações reais) para garantir que o seu impacto e as suas conseqüências em longo prazo não reforcem falsos moralismos, preconceitos e/ou concepções errôneas relacionadas ao tema HIV/DST, incluindo o conhecimento relativo à transmissão vertical.

Cabe destacar a importância da educação em saúde durante o pré-natal para a inserção da gestante nas ações efetivas de prevenção do HIV/DST. A aquisição de conhecimentos pressupõe o desenvolvimento da capacidade para avaliar criticamente ações que irão favorecer a qualidade de vida do binômio mãe-bebê.

Apesar disto, a maioria das gestantes parece não ter assimilado, claramente, diversas informações e/ou conceitos fundamentais para a compreensão 
dos aspectos relacionados ao HIV. Dados incorretos acerca da função e da interpretação do resultado da Sorologia para o HIV, significado do termo "portador do vírus do vírus HIV", bem como a diferença entre os termos "portador assintomático" e a "fase da Síndrome da Imunodeficiência Adquirida (AIDS)", denotaram a ocorrência de falhas durante o processo de assimilação do conhecimento referente ao HIV/AIDS/DST, ocorrido no pré-natal.

Esse exame é para saber se a mulher pode adquirir o vírus ou não. (Gesta 04)

A Sorologia para HIV tem como função a detecção da presença do vírus HIV no organismo humano. A possibilidade de contaminação pelo vírus HIV está diretamente relacionada à não observância das práticas e/ou medidas preventivas relacionadas à transmissão do HIV, não tendo correlação com a realização deste exame.

Outro fato que merece relevância é a dificuldade para identificar a diferença entre a condição de "portador do vírus HIV" (conhecida também como soropositividade) e o início das fases "sintomáticas" de "síndrome da imunodeficiência" provocadas pelo HIV (na qual o indivíduo apresenta "doenças oportunistas" que levam à morte). Para uma gestante esta diferenciação não é percebida, o que pode reforçar preconceitos e redução da capacidade de enfrentamento diante do diagnóstico positivo do HIV.

Esse exame é pra ver se está com a doença HIV. (Gesta 08)

Para uma gestante o vírus HIV não representaria uma ameaça à condição humana. Em seu depoimento esta gestante parece reduzir a possibilidade de transmissão e a virulência do HIV a sua classificação taxonômica, o que deixa claro sua incapacidade para compreender o conceito de "soro positividade" e as suas implicações na saúde do indivíduo e do bebê. Neste caso, esta mulher também associou, de maneira incorreta, a "percepção corporal" como uma estratégia preventiva contra este vírus.
O HIV é só um vírus. Se a gente não conhece o nosso corpo pode pegar a doença. (Gesta 10)

A gravidez é um processo fisiológico que representa a capacidade reprodutiva inerente à mulher, conferindo uma série de mudanças físicas e emocionais, que podem produzir sentimentos e percepções diversas. Neste momento, o desempenho do profissional de saúde torna-se fundamental, uma vez que, deverá contribuir para apoiar, orientar e desenvolver uma relação de confiança que auxiliará o fortalecimento e a condução, de forma mais autônoma, da gestação e do parto. ${ }^{(7)}$

Em mais um depoimento, outra gestante nem mesmo apresenta uma resposta objetiva em relação à função da Sorologia para o HIV, revelando sua postura indiferente durante $\mathrm{O}$ acolhimento/ aconselhamento pré-natal.

Esse exame é pra prevenir qualquer coisa, tipo assim pra que não aconteça nada com o bebê. (Gesta o6)

O acolhimento da mulher durante o pré-natal (desde o início da gravidez) deve permitir o diálogo franco baseado na sensibilidade e na capacidade de percepção de quem faz este acompanhamento. Estas podem ser consideradas condições básicas para que o saber em saúde seja colocado à disposição da mulher e de sua família, bem como elementos chave na construção das práticas preventivas em saúde associadas à prevenção do HIV/DST.

Os principais objetivos que devem ser atingidos durante o Aconselhamento pré/pós-teste HIV/DST relacionam-se a ênfase nos aspectos preventivos, ao estabelecimento de vínculos de confiança, e a promoção dos direitos do cidadão. ${ }^{(8)}$

Durante as práticas educativas realizadas no acolhimento pré-natal, vários conceitos são mencionados. Além disto, é necessário avaliar as condições do ambiente, a adequação da abordagem educativa, bem como a capacidade do profissional para transmitir as informações e permitir a assimilação das mesmas. Seria necessário considerar, também, de que maneira este processo ensino- 
-aprendizagem poderia ser avaliado, o que poderia nortear a continuidade ou a reformulação destas práticas educativas.

No que se refere a estas considerações, outra gestante demonstrou sua insatisfação em relação ao tempo utilizado durante o acolhimento/aconseIhamento pré-natal.

Os aconselhamentos pré e pós-sorologia para o HIV podem ser utilizados para tornar proveitoso o intervalo de tempo entre a espera e a consulta. Esta estratégia pode ser facilitada utilizando-se palestras e outros meios de informação para as gestantes e os seus parceiros, evitando a banalização das informações referentes à realização da Sorologia para HIV. Durante o aconselhamento pós-teste, o profissional de saúde deverá discutir o resultado do exame com a gestante, reforçando os aspectos preventivos do HIV/DST, qualquer que seja o resultado. ${ }^{(10)}$

Ah, eu acho importante porque a gente fica sabendo o que está acontecendo comigo e com o bebê. Mas eu acho muito demorado. (Gesta o6)

A falta de adequação da capacidade instalada nos serviços de saúde e a deficiência no número de profissionais que realizam o aconselhamento podem provocar a massificação do serviço, o receio de não transmitir informações indispensáveis e a baixa valorização desta atividade. ${ }^{(11)}$

Cabe ainda ressaltar a influência psicológica relacionada à realização/interpretação do resultado da Sorologia para HIV, bem como a influência do acolhimento/aconselhamento pré-natal na utilização do preservativo masculino, mesmo que esta utilização não seja freqüente.

É possível questionar, até que ponto estas gestantes (e seus parceiros) estão adotando comportamentos preventivos em relação ao HIV, devido à referência a sensação de "alívio". Se não existissem comportamentos de risco, não deveria haver tanto temor em relação à realização da Sorologia para HIV, apesar dos estigmas que este vírus ainda mantém.
No caso de quem faz o exame e dá positivo é ruim para o seu psicológico. Já pra quem dá negativo é (...) Sei lá! É um alívio! (Gesta 03)

Eu tenho um marido que é bem danado! A gente usa o preservativo, mas nem sempre. Quando eu venho e faço o acompanhamento isso influencia no uso do preservativo. (Gesta 02)

Para que, de fato, o aconselhamento seja efetivo, é necessário que o cliente se aproprie de informações científicas, transformando-as no principal instrumento para adoção de comportamentos com menor risco de transmissão do HIV/DST. ${ }^{(1)}$

Ao procurar o serviço de saúde durante a realização do pré-natal, a gestante pode deparar-se com as dificuldades relacionadas ao atendimento oferecido nos serviços públicos de saúde, que vão desde a dificuldade para marcar as consultas, até a inexistência das condições mínimas preconizadas pelo Ministério da Saúde para a realização da assistência pré-natal. Ao serem detectados estes problemas, há um desencontro entre o que é oferecido pelos serviços e o que a gestante deseja. ${ }^{(12)}$

A verificação da existência de comportamentos de risco coloca em questão a qualidade e a eficácia da atuação profissional durante o acolhimento/aconselhamento pré-natal. Contudo, é necessário ressaltar que o processo ensino-aprendizagem inclui a transmissão do conhecimento através de uma relação recíproca entre multiplicador e o público-alvo. As falhas relacionadas à aquisição do conhecimento e sua conversão em medidas preventivas dependem de muitas variáveis, cujo controle nem sempre está somente nas mãos da equipe de saúde.

É fundamental que haja interesse, por parte das gestantes, para perceber que o conhecimento oferecido durante o acolhimento/aconselhamento pré-natal é fundamental para a prevenção e a manutenção da qualidade de vida da mãe, do bebê e de todo ser humano que possa ser contaminado pelo vírus HIV. 
Dentro deste contexto, a realização da Sorologia para o HIV pode ser considerada como um instrumento que verificaria a aquisição do conhecimento necessário e fundamental para adoção de comportamentos preventivos permanentes e fundamentais para reduzir as taxas de incidência do HIV, inclusive durante a gestação.

\section{CONCLUSÕES}

A instituição selecionada para a realização desta pesquisa apresenta um trabalho de grande expressividade em relação ao acolhimento/aconselhamento pré-natal. $\mathrm{O}$ trabalho é realizado de forma interdisciplinar, rotineiramente. As gestantes são sensibilizadas para a participação, durante o pré-natal, das atividades educativas realizadas no acolhimento/ aconselhamento (que incluem conhecimento/realização da Sorologia para o HIV).

Apesar dos esforços da equipe de saúde, as práticas preventivas que permeiam o acolhimento/ aconselhamento pré-natal, encontram-se, em parte, tolhidas pela demanda excessiva absorvida pelo serviço, pela falta de estrutura física adequada, pela indisponibilidade de tempo (adequado) para promover o processo-ensino aprendizagem referente à infecção pelo Vírus da Imunodeficiência Humana (HIV)/realização da Sorologia para HIV.

A maioria das gestantes apreendeu de forma clara e objetiva os conhecimentos que reforçaram a importância da realização dos exames (inclusive a Sorologia para HIV) e da rotina pré-natal.

Em contrapartida, foram observadas falhas durante a educação em saúde realizada, uma vez que, esta mesma proporção de gestantes não conseguiu apreender de forma clara conceitos que encerram maior complexidade, tais como soro positividade, fase assintomática, tipos de transmissão vertical, entre outros.

É possível considerar que o processo ensinoaprendizagem pode sofrer influência, até mesmo, da motivação do profissional e da gestante. Este tipo de influência pode que interferir negativamente na apreensão do conhecimento durante as práticas educativas associadas à infecção/Sorologia do HIV.

O conhecimento apreendido pela maioria das gestantes acerca da importância da realização Sorologia para o HIV pode ser considerado adequado, uma vez que, estas mulheres referiram a percepção da sua importância para a manutenção da saúde do binômio mãe-bebê, bem como para iniciar tratamento precoce, caso o resultado seja positivo. A maior ênfase nos depoimentos esteve associada à necessidade de evitar a transmissão vertical do HIV durante a gestação.

Foi verificada a utilização da abordagem educativa baseada no "medo". Esta abordagem apresentou como objetivo a conversão do conhecimento em práticas preventivas. É possível considerar que este mesmo sentimento esteja sendo mais associado aos estigmas que envolvem a simples realização da Sorologia para o HIV, o pode induzir a não realização deste exame por algumas gestantes.

A prática preventiva relacionada à transmissão do HIV ainda não é efetivada rotineiramente pela maioria destas gestantes, o que denota que a real transformação do conhecimento em atitudes não foi concluída.

É possível considerar que estas gestantes realizam o pré-natal pela disponibilidade do serviço no Sistema Único de Saúde, bem como pela necessidade de verificação da condição de saúde delas e dos bebês. Porém, em relação à realização da Sorologia para - HIV, apesar de haver conhecimento específico acerca da prevenção e transmissão deste vírus, elas permitem a existência de comportamentos de risco, demonstrados pelo sentimento de "medo" ou "alívio" após a interpretação do resultado deste exame.

$\mathrm{Na}$ verdade parece ser necessária a revisão de competências pessoais destas gestantes para manter sua saúde e, principalmente, garantir a saúde de seus filhos. É desalentador perceber que, atualmente, apesar dos esforços para conter a epidemia do HIV em todos os períodos do ciclo vital humano, ainda existam pessoas que menosprezam a possibilidade de sua transmissão, incluindo a transmissão vertical. 
Muitos fatores envolvem a epidemia do HIV. É importante rever conceitos e atos. Isto é uma questão pessoal, com implicações coletivas. A segurança do bebê está nas mãos da sociedade. E é esta sociedade que precisa adotar a saúde como bem maior, fundamentado em suas próprias atitudes e escolhas.

\section{REFERÊNCIAS}

1. Barroso LMM, Carvalho CML, Galvão MGT. Mulheres com HIV/AIDS: subsídios para a prática de enfermagem. Rev. RENE. 2006;7(2):67-73.

2. Brasil. Guia de Vigilância Epidemiológica/AIDS. 6a. ed. Brasília (DF); 2006.

3. Brasil. Ministério da Saúde. Portaria Técnica Ministerial n. 2.104, de 19 de novembro de 2002. Regulamenta Projeto-Nascer Maternidades. Diário Oficial da União, Brasília, DF, 19 nov. 2002.

4. Pinto TCR. A educação preventiva nas escolas. In: Amaro CM et al. Papel da educação na ação preventiva ao abuso de drogas e às DST/Aids. $3^{a}$ ed. São Paulo: FDE; 1998. p. 43-45. (Série "Idéias", 29)

5. Brasil. Ministério da Saúde. Programa de Humanização do Parto: humanização no prénatal e nascimento. Brasília; 2002.
6. Triviños A. Introdução à pesquisa em ciências sociais. São Paulo: Atlas; 1994.

7. Brasil. Ministério da Saúde. Coordenação de Saúde Materno Infantil. Programa de Assistência Integral à Saúde da Mulher. Assistência Pré-Natal: manual técnico. $3^{a}$ ed. Brasília; 2000.

8. Israel GR. Como é que eu vou dizer para a gestante? Dilemas morais da oferta do teste anti-HIV a gestantes em trabalho de parto: uma contribuição da biolítica (dissertação). Rio de Janeiro: Escola Nacional de Saúde Pública, Fiocruz; 2002.

9. Brasil. Ministério da Saúde. Coordenação de Saúde Materno Infantil. Programa de Assistência Integral à Saúde da Mulher. Assistência Pré-Natal: normas e manuais técnicos. 3a ed. Brasília; 1997.

10. Brasil. Ministério da Saúde. Secretaria de Vigilância em Saúde. Programa Nacional de DST e AIDS. Protocolo para Prevenção de Transmissão Vertical de HIV e Sífilis. Brasília; 2006.

11. Araújo CLF, Camargo Júnior KR. Aconselhamento em DST/HIV: repensando conceitos e prática. Folha Carioca 2004.

12. Marcon SS. "Flashes" de como as gestantes percebem a assistência pré-natal em um hospital universitário. Rev. latinoam. enferm. 1997;5(4):43-54. 